\title{
MOTIVOS PARA EL CONSUMO DE TABACO Y SU RELACIÓN CON LA RESPUESTA AFECTIVA Y LA AUTOEFICACIA EN PROFESIONALES DE LA SALUD
}

\author{
REASONS FOR TOBACCO USE, AND THEIR \\ RELATIONSHIP WITH AFFECTIVE RESPONSE AND SELF- \\ EFFICACY IN HEALTH PROFESSIONALS
}

\author{
Paola Alejandra Vaca Yépez 1 \\ Roberto Javier Enríquez Anaya²
}

Recibido: 2021-03-12 / Revisado: 2021-04-16 / Aceptado: 2021-05-10 / Publicado: 2021-07-01

Forma sugerida de citar: Vaca-Yépez, P. A. y Enriquez-Anaya, R. J. (2021). Motivos para el consumo de tabaco y su relación con la respuesta afectiva y la autoeficacia en profesionales de la salud. Retos de la Ciencia. 5(11), pp. 36-43. https://doi.org/10.53877/rc.5.11.20210701.04

\section{RESUMEN}

El objetivo de esta investigación fue estimar la relación entre las variables motivos para el consumo de tabaco, respuesta afectiva y autoeficacia en los profesionales de la salud del Hospital Gíneco Obstétrico Isidro Ayora en el año 2020. Se realizó un estudio de tipo cuantitativo de corte transversal. Se aplicó la Escala de Motivos para Fumar (Russell), la Escala de Afecto Positivo y Negativo (Watson, Clark y Tellegen) y la Escala de Autoeficacia General (Baessler y Schwarzer), en una muestra de 78 fumadores. Los resultados obtenidos reflejaron que, si existe una relación estadística significativa entre los motivos para el consumo de tabaco y la respuesta afectiva, mientras que no se evidenció relación entre los motivos de consumo de tabaco con la autoeficacia. Se puede concluir que el afecto negativo es mayor en los fumadores por motivos psicológicos y la autoeficacia no parece influir en los motivos para el consumo de tabaco en la población de estudio.

Palabras clave: tabaco, afectividad, autoeficacia, profesionales de la salud.

\section{ABSTRACT}

The objective of this research was to estimate the relationship between the variable's reasons for tobacco consumption, affective response and self-efficacy in the health

\footnotetext{
${ }^{1}$ Magister en Intervención Psicológica en Adicciones. Psicóloga Mención Clínica. Psicóloga Ministerio de Salud Pública del Ecuador - Hospital Gíneco Obstétrico Isidro Ayora. Ecuador. Email: paolavaca11@hotmail.com / ORCID: https://orcid.org/0000-0002-0159-3771

2 Magister en Prevención y Asistencia de las Drogodependencias. Docente de la Maestría de Intervención Psicológica en Adicciones de la Universidad Central del Ecuador. Ecuador. Email: msp.drogodependencias@gmail.com / ORCID: https://orcid.org/0000-0003-0589-3007
} 
professionals of the Hospital Gíneco Obstétrico Isidro Ayora in 2020. A quantitative, cross-sectional study was carried out. The Reasons to Smoke Scale (Russell), the Positive and Negative Affect Scale (Watson, Clark and Tellegen) and the General Self-Efficacy Scale (Baessler and Schwarzer) were applied in a sample of 78 smokers. The results obtained reflected that, if there is a statistically significant relationship between the reasons for smoking and the affective response, there was no evidence of a relationship between the reasons for smoking and self-efficacy. It can be concluded that negative affect is greater in smokers for psychological reasons and self-efficacy does not seem to influence the reasons for tobacco use in the study population.

Key words: tobacco, affectivity, self-efficacy, health professionals.

\section{INTRODUCCIÓN}

El consumo de tabaco es un problema de salud pública que provoca la muerte de la mitad de sus consumidores. Se estima que cada año mueren alrededor de 8 millones de personas debido al consumo directo del tabaco y cerca de 1,2 millones de consumidores pasivos, que son aquellos que no fuman, pero están expuestos de manera involuntaria al humo de los fumadores. Respecto a los 1,300 millones de fumadores que existen en el mundo, el $80 \%$ viven en países con ingresos medios a bajos, en donde la incidencia de mortalidad es más alta que en los países desarrollados (Organización Mundial de la Salud, 2019).

En el Ecuador según la encuesta nacional realizada en estudiantes de secundaria, se identificó que la edad promedio de inicio de consumo de drogas lícitas e ilícitas se da entre los 14 a 15 años, siendo el alcohol y el tabaco las sustancias más consumidas (SETED, 2016); mientras que el estudio epidemiológico andino sobre el consumo de drogas en universitarios, evidenció que los estudiantes ecuatorianos tienen un mayor porcentaje de relación de consumo de alguna vez en la vida de tabaco 54,9\%, en relación con los estudiantes de Perú 52,1\%, Colombia $51,5 \%$ y Bolivia 46,3 \% (Oficina de las Naciones Unidas Contra la Droga y el Delito, 2017).

Existen varios factores determinantes para el inicio y mantenimiento del consumo de tabaco en la población, pero se ha identificado que ciertos factores emocionales como la ansiedad, la depresión y la presencia de afectos negativos pueden ser condicionantes para el consumo (Lira et al., 2018; Moral de la Rubia, 2011). Además, al consumo de tabaco se le asocia como un reforzador psicológico positivo que permite a los consumidores mejorar la atención, memoria, incrementar el nivel de alerta, pero también se conoce que dicho consumo se puede presentar como reforzador negativo, para reducir la ansiedad, tristeza, estrés e irritabilidad, siendo esto un factor determinante para la dependencia, mantenimiento del consumo, problemas en la deshabituación y recaída de los fumadores (American Cancer Society, 2015; Martínez y Becoña, 2016; Redolat, Carrasco y Simón, 1994).

Debido a lo expuesto, se estima que una intervención temprana en la reducción de los problemas emocionales de las personas puede influir de manera directa en la prevención del consumo de tabaco, conociendo además que, una baja percepción de autoeficacia en los fumadores disminuirá su capacidad y habilidad para afrontar situaciones de riesgo y de estrés, lo cual influye en el mantenimiento de la conducta de consumo (Pérez, Sesé, Romo, Palmer y Tomás, 2010; Morales, Díaz, Garrido y Pascual, 2011). 
Desde esta perspectiva, se considera que el consumo de tabaco en profesionales de la salud provoca mayor riesgo, ya que, al ser estos agentes de cambio en beneficio de la salud de los pacientes, cumplen una función modélica, y una baja percepción de riesgo del consumo, reducirá la probabilidad de desalentar esta conducta en sus pacientes (Sánchez y Lisanti, 2003; Zonana y Márquez, 2014). Es por esto por lo que, esta investigación permitió identificar aquellos motivos que indicen en el consumo de tabaco de los profesionales, así como también los niveles de autoeficacia y el tipo de respuesta afectiva predominante, que resultan ser condicionantes para el mantenimiento de esta conducta, siendo esto un aporte para el desarrollo de estrategias de prevención e intervención oportunas, enfocadas en el control emocional y desarrollo de la autoeficacia, para disminuir la conducta de consumo.

\section{METODOLOGÍA}

Estudio cuantitativo correlacional de corte transversal con una muestra de 78 profesionales de la salud que consumen tabaco, cuyas edades oscilan entre los 20 a 69 años, y cuya selección se realizó por medio de un muestreo no probabilístico. Se aplicaron tres escalas para la obtención de la información: Escala de Motivos para Fumar (SMS -18), Escala de Afecto Positivo y Negativo (PANAS), Escala de Autoeficacia General (EAG) y una ficha de datos sociodemográficos, la misma que fue elaborada en relación a la Encuesta Nacional de Salud y Nutrición (ENSANUT, 2014) en donde se considera importante identificar: edad, género, estado civil, número de hijos, profesión, identificación de convivencia familiar, entre otros y respecto al consumo de tabaco se preguntó la edad de inicio de consumo, la frecuencia de consumo, la cantidad de consumo diario y la marca de tabaco que usualmente consumen.

La escala de escala de Motivos para Fumar SMS-18 consta de 18 preguntas y fue elaborada por Russell (1979) con el objetivo de identificar los motivos por los cuales las personas consumen tabaco, siendo estos: motivos psicológicos (preguntas 1, 2, 3, 4, 5 y 6), motivos sociales (preguntas 7, 8, 9, 10, 11 у 12) у motivos físicos (preguntas 13, 14, 15, 16, 17 y 18). Esta escala no ha sido aplicada en población ecuatoriana, por lo que se realizó una adaptación lingüística con el apoyo de profesionales que convocó la Secretaría del Comité Interinstitucional de Prevención Integral del Fenómeno Socio Económico de las Drogas y de Regulación y Control del Uso de Sustancias Catalogadas Sujetas a Fiscalización del Ecuador. Dichos profesionales realizaron la validación en relación a la claridad de las instrucciones de la escala, la claridad del contenido de los ítems y el formato de la misma. Con las observaciones de los expertos y acogiendo sus sugerencias se realizó un pilotaje de la escala, la cual presentó un coeficiente de 0.89 Alpha de Cronbach.

La escala de Afecto Positivo y Negativo (PANAS) es una escala auto aplicada que mide la afectividad positiva y negativa; fue creada por Watson, Clark, y Tellegen (1988) y está estructurada por 20 adjetivos que describen emociones (10 positivas y 10 negativas. La escala (PANAS) ha sido validada en población ecuatoriana por García y Arias (2019). En esta investigación la escala presentó un coeficiente 0.83 de Alpha de Cronbach.

Para la evaluación de la autoeficacia se utilizó la Escala de Autoeficacia General (EAG) que fue traducida al español por Baessler y Schwarzer (1996) y adaptada a la 
población ecuatoriana por Bueno, Lima, Peña, Cedillo y Aguilar (2018), la misma que contiene 10 ítems con frases sobre la confianza que las personas tienen para enfrentarse a diferentes situaciones, fundamentándose en el concepto de autoeficacia de Bandura. La Escala de Autoeficacia General, presentó 0.94 de coeficiente de Alpha de Cronbach.

Para el procesamiento de datos y obtención de resultados se utilizó el software SPSS en su versión 16. Los resultados de los datos descriptivos se obtuvieron por medio de tablas de frecuencia y de porcentajes y por medio de la prueba estadística $\mathrm{Chi}^{2}$ se evaluó la relación de las variables del estudio.

\section{RESULTADOS}

\section{Datos descriptivos}

La investigación se realizó con 78 profesionales de la salud que consumen tabaco, de los cuales el $59 \%$ fueron de sexo masculino y el $41 \%$ de sexo femenino, cuyas edades evidenció que 2 de cada 10 tenían edades comprendidas entre 22 a 27 años, 28 a 33 años y 34 a 39 años, en referencia a 1 de cada 10 que tenían edades entre 40 a 45 años, 46 a 51 años, 52 a 57 años y 58 a 66 años, siendo el rango de edad mayormente representado el de edades comprendidas entre 34 a 39 años en comparación a los otros grupos etarios.

En relación con la edad de inicio de consumo de tabaco se obtuvo una media de 16,85 años. Respecto a la frecuencia del consumo de tabaco, el $33,3 \%$ de los encuestados se consideran fumadores diarios, el $28,2 \%$ fumadores semanales, $10,3 \%$ fumadores ocasionales (cada 15 días) y finalmente el $28,2 \%$ refirieron fumar una vez al mes, siendo la marca Lark $47,4 \%$ la que más consumen.

Los resultados sobre los motivos para el consumo de tabaco reflejaron que el $64,1 \%$ de los encuestados fuman por motivos psicológicos, el $26,9 \%$ por motivos físicos y el $9 \%$ por motivos sociales. Respecto a los motivos psicológicos se refiere al consumo como un medio para aumentar la confianza social, disminuir los afectos negativos y mantener una sensación de control del estrés (Russell, 1971a).

La respuesta afectiva predominante en los fumadores fue en el 76,9\% afecto positivo y $23,1 \%$ afecto negativo. En relación con los motivos de consumo de tabaco y la respuesta afectiva se pudo conocer que, de los fumadores por motivos psicológicos, el $51,3 \%$ presentaron afectividad positiva y $12,8 \%$ afectividad negativa. En los fumadores por motivos físicos el $16,7 \%$ presentaron afectividad positiva y el $10,3 \%$ afectividad negativa y en los fumadores por motivos sociales el $9 \%$ presentaron afectividad positiva y no se evidenció afectividad negativa.

Los resultados sobre los niveles de autoeficacia reflejaron que el $83,3 \%$ de los encuestados presentan autoeficacia alta y el 16,7\% niveles bajos de autoeficacia. Respecto a los niveles de autoeficacia y los motivos de consumo de tabaco, de los consumidores por motivos psicológicos 6 de cada 10 presentaron autoeficacia alta y 1 de cada 10 autoeficacia baja, mientras que en los consumidores por motivos físicos 2 de cada 10 presentaron autoeficacia alta y 1 de cada 10 autoeficacia baja, finalmente, todos los consumidores por motivos sociales presentaron autoeficacia alta.

\section{Estadística inferencial}

Para el análisis de las variables de este estudio se utilizó la prueba Chi ${ }^{2}$. Respecto a los resultados de los motivos de consumo de tabaco y su relación con la respuesta afectiva se obtuvo un $\left(X^{2}=2,56 ; p<0,05\right)$ por lo que se estima que si existe relación 
entre los motivos para el consumo de tabaco y la respuesta afectiva, mientras que con las variables de autoeficacia y motivos de consumo de tabaco se obtuvo un resultado de $\left(X^{2}=4 ; p>0,05\right)$ por lo que se establece que no existe relación entre los motivos de consumo de tabaco con los niveles de autoeficacia en los profesionales de la salud.

\section{DISCUSIÓN}

El objetivo de esta investigación realizada en profesionales de la salud fue analizar la relación entre los motivos de consumo de tabaco, la respuesta afectiva y la autoeficacia, por lo que se efectuó la aplicación empírica de los conceptos motivos para el consumo de tabaco (Russell, 1979), afectividad (Watson, Clark y Tellegen, 1988) y autoeficacia (Bandura, 2006).

El estudio se realizó con una muestra de 78 profesionales de la salud de edades comprendidas entre los 22 a 66 años, con una media de edad de 39,03 años, que resultó ser similar a la de otras investigaciones realizadas con personal de salud en donde se reportó una edad madura de los encuestados (Sánchez, 2009; Sánchez, 2010). De los profesionales el $59 \%$ fueron de sexo masculino y el $41 \%$ de sexo femenino, tal como se ha presentado en otras investigaciones (Moreno y Herrero, 2000; Peña y Esparza, 2017; Sánchez, 2010).

En relación a los motivos psicológicos para el consumo de tabaco el 15,4\% reportaron que casi siempre y siempre fuman cuando se sienten frustrados, el $16,7 \%$ indicaron que siempre y casi siempre fuman cuando se sienten enojados, el $19,3 \%$ refirieron que siempre y casi siempre fumar les relaja cuando están tensos, el $20,5 \%$ casi siempre y siempre fuman cuando están enojados por algo o con alguien, siendo estos resultados similares a los de otros estudios realizados con personal de salud (Sánchez, 2010). Dichos hallazgos apoyan el enfoque relacionado a la conducta del consumo de tabaco como un medio para mitigar el afecto negativo, siendo los reforzadores positivos y negativos determinantes en el mantenimiento de las conductas adictivas (Lira et al., 2020; Pormeleau, Fagerstrom, Marks, Tate, 2003).

Asimismo, se pudo identificar que la presencia de afecto negativo se presenta con mayor prevalencia en los fumadores por motivos físicos y psicológicos, lo cual permite inferir que estos motivos se relacionan con la presencia de emociones negativas y que por medio del consumo de tabaco se puede lograr una regulación de dichos estados, a fin de reducir la tensión o por la necesidad de consumo asociada a los síntomas de dependencia física, siendo estos resultados un aporte a la teoría de Russell (1971b), en donde se plantea que los motivos psicológicos para fumar se dan en función de disminuir las emociones negativas; además, dichos resultados se han evidenciado en investigaciones realizadas con fumadores diarios de tabaco, en donde se reporta que la sensibilidad a la ansiedad genera mayor motivación para fumar en fin de reducir el afecto negativo (Johnson, Farris, Schmidt, Smits y Zvolensky, 2013).

En comparación a las investigaciones realizadas por Leal et al., (2010) en una población de fumadores que acudían a un tratamiento para dejar de fumar y en el estudio realizado por Martín (2016), sobre lo factores asociados a la cesación tabáquica en pacientes de una unidad de deshabituación, se pudo identificar que la presencia de sintomatología depresiva en los fumadores aumenta la probabilidad de recaída en el consumo, mientras que la presencia de síntomas de estrés o 
ansiedad, disminuye la probabilidad de llegar a una abstinencia. Desde esta perspectiva se puede inferir que los afectos negativos influyen en el mantenimiento del consumo de tabaco y en los procesos de recaída cuando se ha logrado una abstinencia.

Referente a los resultados del estudio regulación emocional, afectividad y razones para fumar en fumadores diarios de tabaco, realizado por Navarro y Musso (2016), se identificó que existe una asociación entre los motivos para el consumo de tabaco y el estado afectivo negativo, lo cual permitió determinar que, a mayor afecto negativo, se desarrollan mayores razones para fumar vinculadas a patrones de dependencia y adicción.

Acerca de los resultados obtenidos sobre la variable niveles de autoeficacia percibida y los motivos de consumo de tabaco, no se encontró una correlación estadística significativa al realizar la prueba $\mathrm{Chi}^{2}$, por lo que se estima que no es necesario presentar motivos físicos y psicológicos de consumo de tabaco para que existan niveles bajos de autoeficacia. En esta línea, se concluye que los motivos para el consumo de tabaco físico y psicológicos no parecen ser un factor que influye en la autoeficacia de los profesionales de la salud que consumen tabaco, resultados similares se han obtenido en población universitaria, en donde se aprecia que el estudiar representa un factor protector y que los niveles altos de autoeficacia encontrados, podrían deberse a que los universitarios se encuentran en un proceso de formación en donde deben enfrentarse a diferentes situaciones que les permite desarrollar habilidades (Godoy y Medel, 2012), lo cual podría resultar similar a la condición de los profesionales de la salud.

\section{CONCLUSIONES}

Los resultados permiten concluir que, si existe una relación estadística significativa entre los motivos para el consumo de tabaco y la respuesta afectiva, mientras que no se evidenció relación entre los motivos de consumo de tabaco y la autoeficacia.

Los motivos psicológicos para fumar predominaron en los profesionales de la salud, lo cual permite inferir que dicho consumo probablemente se da para disminuir el cansancio, la fatiga, modular la presencia de afectos negativos y provocar una sensación de control del estrés.

Finalmente se evidenció, que la muestra mayoritaria del estudio estuvo representada por fumadores diarios de tabaco, fumadores semanales y de una vez al mes, por lo que se estima que los profesionales encuestados no se encuentran en una fase de experimentación del consumo, sino que existe una relación con esta sustancia que podría generar patrones de dependencia y riesgo para su salud; además, los profesionales que muestran motivos físicos para el consumo de tabaco presentaron mayor cantidad de consumo, lo cual permite inferir que los motivos para fumar por reforzamiento negativo se relacionan con niveles de dependencia a la nicotina.

\section{REFERENCIAS}

American Cancer Society. (2015, noviembre 24). Por qué la gente comienza a fumar y por qué es difícil dejar el hábito ¿Por qué las personas comienzan a fumar? Recuperado 17 de septiembre de 2020, de https://www.cancer.org/es/saludable/mantengasealejado-del-tabaco/por-que-la-gente-comienza-a-fumar.html. 
Baessler, J., y Schwarzer, R. (1996). Evaluación de la autoeficacia: Adaptación española de la escala de Autoeficacia General. Ansiedad y Estrés, 2, 1-8.

Bandura, A. (2006). Guide for constructing self-efficacy scales. En F. Pajares y Urdan, T. (Ed), Self-efficacy beliefs of adolescence (pp. 307-37). Greenwich: Information Age Publishing.

Bueno, A., Lima, S., Peña, E., Cedillo, C., y Aguilar, M. (2018). Adaptación al español de la escala de autoeficacia general para su uso en el contexto ecuatoriano. Revista Iberoamericana de Diagnóstico y Evaluación Psicológica, 3(48), 5-17. doi: 10.21865/ridep48.301.

García, F., y Arias, P. (2019). Propiedades psicométricas de la Escala de afecto positivo y negativo en población ecuatoriana. Revista Mexicana de Psicología, 36(1), 55-62.

Godoy, P., y Medel, M. (2012). Autoeficacia y su relación con el consumo de tabaco y alcohol en estudiantes universitarios (tesis de pregrado). Universidad del Bío-Bío, Chillán, Chile.

Johnson, K.A., Farris, S.G., Schmidt, N. B., Smits, J. A.., y Zvolensky, M. J. (2013). Panic attack historu and anxiety sensitivity in relation to cognitive - based smoking processes among treatment - seeking daily smokers. Nicotine and Tobacco Research, 15(1), 1-10.

Leal, B., Ocampo, M., y Cicero, R. (2010). Niveles de asertividad, perfil sociodemográfico, dependencia a la nicotina y motivos para fumar en una población de fumadores que acude a un tratamiento para dejar de fumar. Salud Mental, 33(6), 489-497.

Lira, J., Valdéz, E., Núñez, M., Pech, D., Morales, S., y Cruz, S. (2018). Ansiedad, depresión, afecto negativo y positivo asociados con el consumo de tabaco en universitarias. Revista Internacional de Investigación en Adicciones, 4(1), 4-12.

Lira, J., Vald\{ez, E., Pérez, G., Pech, P., y Arriaga, R. (2020). Asociación entre consumo de tabaco, factores emocionales y motivos de fumar en universitarios. Enseñanza $e$ Investigación en psicología, 2(1), 47-59.

Martín, F. (2016). Factores asociados con la cesación tabáquica en los pacientes atendidos en la Unidad de Deshabituación Tabáquica del Servicio Cántabro de Salud (tesis de doctorado). Universidad de Cantabria, Santander, España.

Martínez, C., y Becoña, E. (2016). La sensibilidad a la ansiedad y el consumo de tabaco: Una revisión. Ansiedad y estrés, 22(2). 118-122. doi:10.1016/j.anyes.2016.10.005.

Ministerio de Salud Pública del Ecuador e Instituto de Nacional de Estadísticas y Censos (2014). Encueta Nacional de Salud y Nutrición ENSANUT. Recuperado de https://www.ecuadorencifras.gob.ec/documentos/webinec/Estadisticas_Sociales/ENS A NUT/MSP_ENSANUT-ECU_06-10-2014.pdf

Moral de la Rubia, J. (2011). La escala de afecto positivo y negativo (PANAS) en parejas casadas mexicanas. Ciencia ergo-sum, 18(2), 117-125.

Morales, Z., Díaz, C., Garrido, M., y Pascual, L. (2011). Autoeficacia y consumo de tabaco en estudiantes universitarios. Apuntes de psicología, 29(3), 459-470.

Moreno, J., y Herrero, F. (2000). Factores asociados al éxito en programas para dejar de fumar. Adicciones, 12(3), 329-341.

Navarro, M., y Musso, M. (2016). Regulación emocional, afectividad y razones para fumar: un estudio en adultos fumadores diarios de tabaco (tesis de pregrado). Universidad Argentina de la Empresa. Buenos Aires, Argentina.

Oficina de las Naciones Unidas Contra la Droga y el Delito. (2017). III Estudio epidemiológico andino sobre consumo de drogas en la población universitaria, Informe Regional, 2016. Recuperado de https://www.unodc.org/documents/peruandecuador//Informes/Otros/Informe_Universi tario_Regional.pdf

Organización Mundial de la Salud. (2019). Tabaco. Recuperado 27 de mayo de 2020, de https://www.who.int/es/news-room/fact-sheets/detail/tobacco 
Peña, G., y Esparza, S. (2017). Alcohol y tabaco: Dependencia, motivos, desordenes y consumo en estudiantes Mazatlecos. Revista Conjetura Sociológicas, 5(13), 142174.

Pérez, F., Sesé, A., Romo, A., Palmer, y Tomás, E, (2010). Influencia de las Emociones Negativas (Ansiedad, Depresión e Ira) sobre la Eficacia de un Programa de Tratamiento Cognitivo Conductual de Deshabituación al Tabaco. Clínica y Salud, 21(1), 9-19.

Pormeleu, O.F., Fagerstrom, K.O., Marks, J.L., Tate, J.C., y Pormeleau, C. S. (2003). Development and validation of a self-rating scale for positive and negative reinforcement smoking: The Michigan nicotine reinforcement questionnaire. Nicotine and Tobacco Research, 5(5), 711-718.

Redolat, R., Carrasco, M., y Simón, V. (1994). Efectos cognitivos de la nicotina y el tabaco en sujetos humanos. Psicothema, 6(1), 5-20.

Russell, M. (1971a). Cigarette Dependence: I-Nature and Classification. British Medical Journal, 2(57), 330-331.

Russell, M. (1971b). Cigarette smoking: natural history of a dependence disorder. British Journal of Medical Psychology, 44(1), 1-16.

Russell, M. (1979). Nicotine intake and its regulation. Journal of Psychosomatic Research, 24(2), 253-264.

Sánchez, J. (2009). Motivaciones para el consumo de tabaco y alcohol en trabajadores de la salud (tesis de pregrado). Universidad Autónoma de Nuevo León, Nuevo León, México.

Sánchez, L. (2010). Motivaciones para el consumo de tabaco y consumo de tabaco en trabajadores de la salud (tesis de pregrado). Universidad Autónoma de Nuevo León, Nuevo León, México.

Sánchez, P., y Lisanti, N. (2003). Prevalencia de tabaquismo y actitud hacia este hábito entre médicos del Azuay, Ecuador. Revista Panamericana de Salud Pública, 14(1). $1-8$.

Secretaria Técnica de Prevención Integral de Drogas. (2016). Encuesta Nacional sobre Uso y Consumo de Drogas 2016 - Estudiantes de noveno de educación general básica, primero y tercero de bachillerato. Ecuador: SETED.

Watson, D., Clark, L. A., y Telleguen, A. (1988). Development and Validation of Brief Measures of Positive and Negative Affect: The PANAS Scales. Journal of Personality and Social Psychology, 54(6), 1063-1070.

Zonana, A., y Márquez, O. (2014). Nivel de dependencia al tabaco en enfermera y médicos de un hospital general regional. Revista Iberoamericana de educación e investigación en enfermería, 4(1), 45-51. 